Portland State University

PDXScholar

\title{
Passive Bottom Reflection-Loss Estimation Using Ship Noise and a Vertical Line Array
}

\author{
Lanfranco Muzi \\ Portland State University, muzi@pdx.edu \\ Martin Siderius \\ Portland State University \\ Christopher M. Verlinden \\ University of California - San Diego
}

Follow this and additional works at: https://pdxscholar.library.pdx.edu/ece_fac

Part of the Acoustics, Dynamics, and Controls Commons, and the Electrical and Computer

Engineering Commons

Let us know how access to this document benefits you.

\section{Citation Details}

Muzi, L., Siderius, M., \& Verlinden, C. M. (2017). Passive bottom reflection-loss estimation using ship noise and a vertical line array. The Journal of the Acoustical Society of America, 141(6), 4372-4379.

This Article is brought to you for free and open access. It has been accepted for inclusion in Electrical and Computer Engineering Faculty Publications and Presentations by an authorized administrator of PDXScholar. Please contact us if we can make this document more accessible: pdxscholar@pdx.edu. 


\section{Passive bottom reflection-loss estimation using ship noise and a vertical line array}

Lanfranco Muzi and Martin SideriusChristopher M. Verlinden

Citation: The Journal of the Acoustical Society of America 141, 4372 (2017); doi: 10.1121/1.4985122

View online: http://dx.doi.org/10.1121/1.4985122

View Table of Contents: http://asa.scitation.org/toc/jas/141/6

Published by the Acoustical Society of America

\section{Articles you may be interested in}

Detection of an undersea acoustic communications network by an energy detector

The Journal of the Acoustical Society of America 141, 4136 (2017); 10.1121/1.4984102

Sound Propagation Through the Stochastic Ocean

The Journal of the Acoustical Society of America 141, 4249 (2017); 10.1121/1.4984019

A Marchenko equation for acoustic inverse source problems

The Journal of the Acoustical Society of America 141, 4332 (2017); 10.1121/1.4984272

Vertical line array measurements of ambient noise in the North Pacific

The Journal of the Acoustical Society of America 141, 1571 (2017); 10.1121/1.4976706

Broadband classification and statistics of echoes from aggregations of fish measured by long-range, midfrequency sonar

The Journal of the Acoustical Society of America 141, 4354 (2017); 10.1121/1.4983446

Low frequency acoustic properties of Posidonia oceanica seagrass leaf blades

The Journal of the Acoustical Society of America 141, EL555 (2017); 10.1121/1.4984045 


\title{
Passive bottom reflection-loss estimation using ship noise and a vertical line array
}

\author{
Lanfranco Muzia) and Martin Siderius \\ Department of Electrical and Computer Engineering, Portland State University, 1900 SW 4th Avenue, \\ Portland, Oregon 97201, USA \\ Christopher M. Verlinden \\ Marine Physical Laboratory, Scripps Institution of Oceanography, University of California San Diego, \\ 9500 Gilman Drive, La Jolla, California 92093, USA
}

\begin{abstract}
(Received 17 January 2017; revised 18 May 2017; accepted 19 May 2017; published online 13 June 2017)

An existing technique for passive bottom-loss estimation from natural marine surface noise (generated by waves and wind) is adapted to use noise generated by ships. The original approach-based on beamforming of the noise field recorded by a vertical line array of hydrophones-is retained; however, additional processing is needed in order for the field generated by a passing ship to show features that are similar to those of the natural surface-noise field. A necessary requisite is that the ship position, relative to the array, varies over as wide a range of steering angles as possible, ideally passing directly over the array to ensure coverage of the steepest angles. The methodology is illustrated through simulation and applied to data from a field experiment conducted offshore of San Diego, CA in 2009. (C) 2017 Acoustical Society of America. [http://dx.doi.org/10.1121/1.4985122]
\end{abstract}

[SED]

Pages: $4372-4379$

\section{INTRODUCTION}

The bottom reflection loss can be an important contributor to the total transmission loss, and, especially in shallow waters, its incorrect estimation has been shown to be a major source of error for SONAR performance prediction. ${ }^{1}$ This article proposes a technique for passive estimation of the sea bottom reflection loss by means of a (fixed) vertical line array (VLA), using ship noise as a source of opportunity.

Ships have been used as sources of opportunity for numerous applications, among which geoacoustic inversion is probably the one closest in scope to what is presented in this article. ${ }^{2-8}$ While inversion is often assumed as a preliminary step, to infer the bottom reflection coefficient (and therefore the associated loss), the approach described in this study estimates the bottom reflection loss directly, as a function of frequency and grazing angle, without using an inversion scheme. At the basis of the proposed approach is a technique, presented in 2002 by Harrison and Simons, which has been shown to provide estimates of the bottom loss as a function of frequency and grazing angle, when the field recorded by the array is generated by wind and breaking waves at the surface. ${ }^{9-12}$ The possibility of dispensing with active sound sources makes the technique particularly attractive, because of the reduced environmental impact, equipment complexity, and power consumption. ${ }^{10,13}$ However, the technique can produce inaccurate results in conditions of low wind and/or waves, ${ }^{14}$ and whenever the natural-noise field is contaminated by an interferer, such as the engine of a nearby ship. ${ }^{15}$ It is shown in this study that ships can actually be used as sources of opportunity, to obtain passive estimates

${ }^{a)}$ Electronic mail: muzi@pdx.edu of the bottom reflection loss with Harrison and Simons's technique. This permits the use of the technique in the presence of a nearby ship, and expands its application to those scenarios where the natural surface-noise level is too low to guarantee a reliable estimate. These benefits are achieved by means of a technique for preprocessing the noise field generated by a ship - approaching the array from a distance, and passing over the array or in its immediate vicinity-in such a way as to make it amenable to bottom-loss estimation through Harrison and Simons's technique. The remainder of the article is organized as follows: Section II provides a summary of the basic theoretical treatment of beamforming, and its use in Harrison and Simons's technique; Sec. III illustrates the approach proposed in this article and explains the differences with respect to the original technique with the aid of simulations; Sec. IV shows and discusses the results of the proposed technique on experimental data from the Noise Experiment of $2009,{ }^{16,17}$ and Sec. V summarizes the main findings of the study.

\section{BACKGROUND}

Bottom loss (BL) is defined as a function of the planewave power reflection coefficient $R\left(\theta_{b}, \omega\right)$ as ${ }^{18}$

$$
\mathrm{BL}\left(\theta_{b}, \omega\right)=-10 \log _{10} R\left(\theta_{b}, \omega\right),
$$

where $\omega$ is the angular frequency of the wave front incident upon the bottom at grazing angle $\theta_{b}>0$. In Harrison and Simons's technique, the power reflection coefficient is estimated by applying conventional beamforming (CBF) to the data recorded by a VLA, and computing the ratio of the downward and upward beam powers 9 


$$
\hat{R}\left(\theta_{b}, \omega\right)=\frac{\hat{B}\left(-\left|\theta_{r}\right|, \omega\right)}{\hat{B}\left(\left|\theta_{r}\right|, \omega\right)},
$$

where $\theta_{r}$ is the angle at the receiver, i.e., the angle at which a ray reaches the receiver (see Fig. 1 for a definition of the geometry of the problem). The average beam power $B(\vartheta, \omega)$ of the beamformer is defined as

$$
B(\vartheta, \omega)=E\left[\mathbf{w}^{\mathrm{H}} \mathbf{p}\left(\mathbf{w}^{\mathrm{H}} \mathbf{p}\right)^{\mathrm{H}}\right]=\mathbf{w}^{\mathrm{H}} E\left[\mathbf{p} \mathbf{p}^{\mathrm{H}}\right] \mathbf{w},
$$

where, for the sake of simplicity, the dependence on frequency and angle has been dropped in the right-hand side of the equation (this will be the convention in the remainder of this article). In Eq. (3), $E[\cdot]$ denotes expectation, and $H$ denotes the conjugate transpose operation. $\mathbf{w}(\vartheta, \omega)$ $=\left[w_{1}, w_{2}, \ldots, w_{M}\right]^{T}$ (T denotes the transpose operation) is the weight vector corresponding to the beamformer steering angle $\vartheta$, here defined in such a way that $\vartheta=0$ corresponds to broadside (i.e., steering the array horizontally, in the case of a VLA), $\vartheta>0$ corresponds to steering toward the surface, and $\vartheta<0$ toward the bottom. $\mathbf{p}(\omega)=\left[p_{1}(\omega), p_{2}(\omega), \ldots\right.$, $\left.p_{M}(\omega)\right]^{T}$ is a vector of data recorded by the array, whose $m$ th element is $p_{m}(\omega)=p\left(\mathbf{r}_{m}, \omega\right)=\mathscr{F}\left\{p_{m}(t)\right\}$, where the position vector $\mathbf{r}_{m}$ identifies the location in space of the element, and $\mathscr{F}\{\cdot\}$ denotes the Fourier transform.

The remainder of this article will focus on the expected value of the outer product $\mathbf{p}(\omega) \mathbf{p}^{H}(\omega)$, i.e., the $\mathbf{C}_{\omega}=E\left[\mathbf{p} \mathbf{p}^{H}\right]$ matrix, referring to it as cross-spectral-density matrix (or "CSD matrix" for brevity). The unnormalized spatial coherence function of the pressure field $p(\mathbf{r}, t)$ between the two sensors located at $\mathbf{r}_{i}$ and $\mathbf{r}_{j}$, i.e., the ensemble average:

$$
C_{\omega}\left(\mathbf{r}_{i}, \mathbf{r}_{j}\right) \equiv\left\langle p_{i}(\omega) p_{j}^{*}(\omega)\right\rangle
$$

(where $*$ indicates complex conjugate) gives the generic element $(i, j)$ of $\mathbf{C}_{\omega}$.

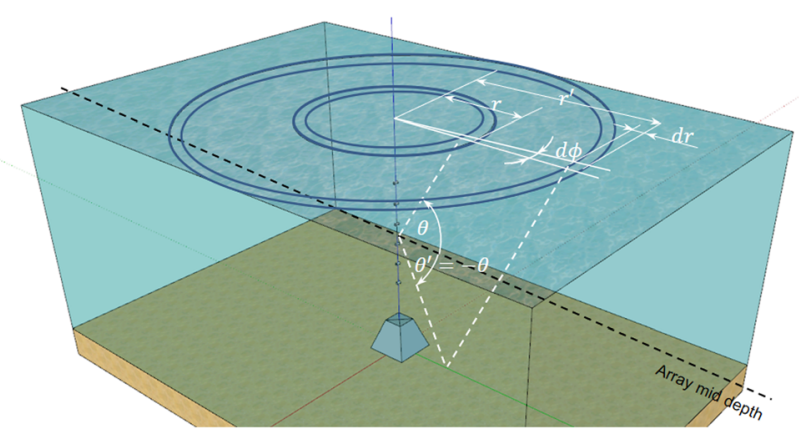

FIG. 1. (Color online) Schematic of Harrison and Simons's technique and definition of reference and geometric quantities showing a moored sixelement array, the bottom, and the sea surface. The array is steered toward two angles that are symmetric with respect to the horizontal. The ellipses show the annular surface elements from which a hypothetical beam of width $d r$ would harvest energy. In Harrison and Simons's original technique, the difference in surface area between the two patches compensates the effect of spreading loss along the two different paths to the array receivers. In the case of a ship being targeted at two different positions $(r, \theta)$ and $\left(r^{\prime},-\theta\right)$, the difference in spreading loss along the two paths must be accounted for. Although a constant-sound-speed water column is assumed in this schematic for simplicity (therefore the ray paths are straight lines), the technique can also be applied in the presence of a sound-speed profile.
While Eqs. (3) and (4) provide theoretical definitions that assume perfect knowledge of the pressure-field statistics, in practice only an estimate $\hat{\mathbf{C}}_{\omega}$ of the CSD matrix can be obtained from measured data, by dividing the time histories at the sensors into $N$ data segments, and averaging the outer product $\mathbf{p}_{n}(\omega) \mathbf{p}_{n}^{H}(\omega)$,

$$
\hat{\mathrm{C}}=\frac{1}{N} \sum_{n=1}^{N} \mathbf{p}_{n} \mathbf{p}_{n}^{\mathbf{H}} .
$$

It is important to note that in a field generated solely by wind and breaking waves at the surface, $C_{\omega}\left(\mathbf{r}_{i}, \mathbf{r}_{j}\right)$ has been shown to be (approximately) spatially stationary, ${ }^{19-22}$ which implies that, besides being Hermitian by construction, $\mathbf{C}_{\omega}$ is also (approximately) Toeplitz. ${ }^{15}$ In general, the spatial stationarity (and the quasi-Toeplitz structure of the CSD matrix) does not hold when the field includes a discrete source at the surface, such as a ship. ${ }^{15}$

\section{METHOD}

\section{A. The "Noise Experiment 2009"}

All the experimental data utilized in this article were acquired during the Noise Experiment of $2009^{16,17}$ (hereafter referred to as "Noise 09" for brevity). The experiment was organized by the Scripps Institution of Oceanography of the University of California, San Diego, between January 30 and February 10 of 2009. During the experiment, four VLAs ${ }^{23}$ were deployed in $150 \mathrm{~m}$ water on the Coronado Bank, approximately $20 \mathrm{~km}$ southwest of Point Loma (San Diego, CA), each comprising 16 elements with an inter-element spacing of $1 \mathrm{~m}$ (corresponding to a design frequency of $750 \mathrm{~Hz}$, at a sound speed of $1500 \mathrm{~m} / \mathrm{s}$ ). The data sampling rate was $25 \mathrm{kHz}$. The arrays were moored to the bottom, with the deepest element about $7 \mathrm{~m}$ above the seabed. The wind speed during the experiment was reported to vary between 0 and $14 \mathrm{~m} / \mathrm{s}$, and the wave height between about 0.5 and $2.3 \mathrm{~m}$. The data shown in this article were all collected by the array identified as "VLA1.,"16,17

\section{B. Effect of discrete sources and low noise levels on the bottom-loss estimate}

The approach described in this study retains Harrison and Simons's application of the CBF steered to symmetric angles to estimate the bottom loss as in Eq. (2) but proposes using a moving ship, rather than natural surface noise generated by wind and waves, as a source of opportunity. The proposed source openly violates one of the assumptions at the basis of the technique-namely, that the field be generated by a sheet of incoherent point sources at the surface. In such cases, the spatial stationarity of the coherence function is not guaranteed, ${ }^{19,20}$ and the CSD matrix does not have a Toeplitz structure. ${ }^{15}$

As an example, Fig. 2 shows the beamformer output and real part of the normalized CSD matrix at $433 \mathrm{~Hz}$, estimated from data recorded by VLA1 around 5:00 and 7:10 UTC on February 6 . Since the array location did not change between the two measurements, the marked difference in the plots 

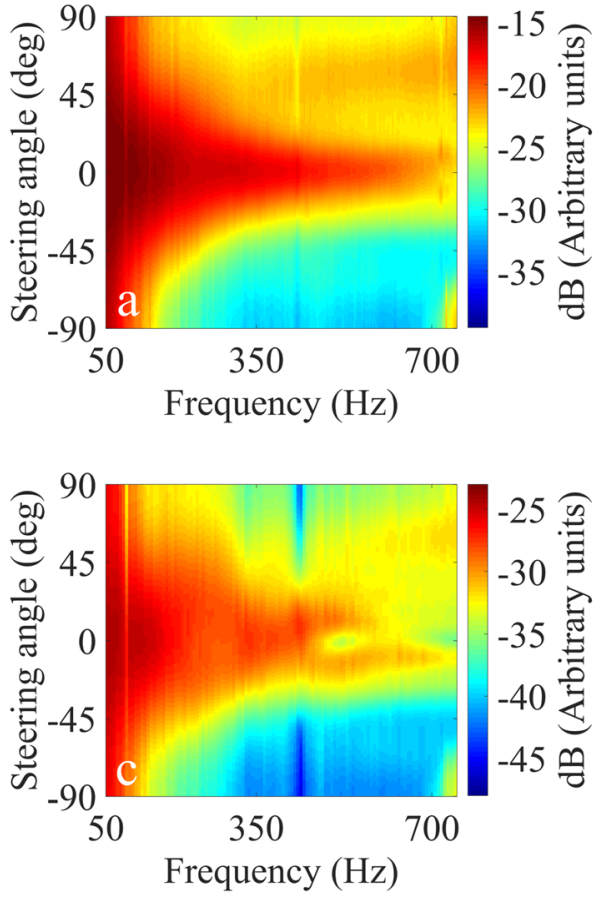
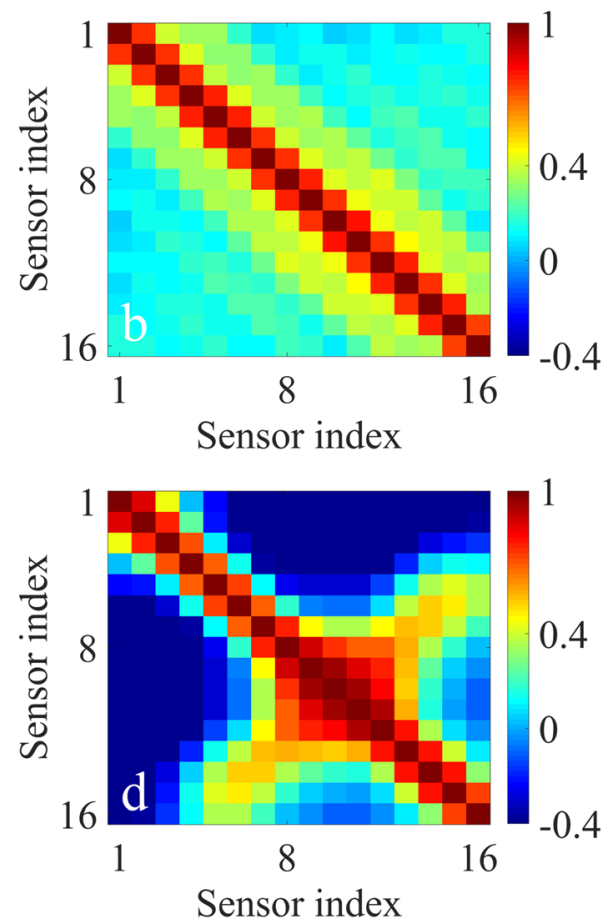

FIG. 2. (Color online) Noise 09, data from February 6: Beamformer output and real part of the normalized CSD matrix at $433 \mathrm{~Hz}$, estimated from a field generated by wind noise in the absence of close interferers [(a) and (b)], and in the presence of interference [(c) and (d)]. Note that the (approximate) Toeplitz structure, displayed by the CSD matrix in the former case, is destroyed in the latter case. cannot be due to a change in the bottom properties, such as could occur in the case of a drifting array. As the beamformer output shows, the data that produced Figs. 2(a) and 2(b) were collected in a field generated by natural noise at the surface (note the diffuse, higher levels in the upper half of the plot), in the absence of close interferers, whereas interference was present during the collection of the data used to produce Figs. 2(c) and 2(d). Note that the (approximate) Toeplitz structure, displayed by the CSD matrix in the former case [Fig. 2(b)], is destroyed by the interferer in the latter case [Fig. 2(c)].

When the spatial stationarity of the coherence function is violated, it has been shown that the ratio in Eq. (2) does not represent the bottom reflection coefficient, and the bottom-loss estimate is not reliable. ${ }^{15}$ Given the ubiquitous nature of ship traffic, this poses a limitation to the applicability of a technique that would be quite attractive, given its simplicity, low environmental impact, and cost effectiveness. Furthermore, in situations of low sea state, the natural noise level generated at the surface may be inadequate to produce an estimate of the reflection loss. ${ }^{14}$ This is shown in Fig. 3, where bottom-loss estimates obtained from data corresponding to low and moderate sea state are compared, showing in the former case a very significant underestimation of the loss. The approach proposed in this study consists of preprocessing the data recorded while a ship moves over a range of distances from the array (from several kilometers to possibly passing over the array), so that it can be exploited as a source, to aid the bottom-loss estimation, even in such "extreme" cases as the one shown in Fig. 3(a).

The result in Fig. 3(b) deserves some attention, as it will be used as reference for the rest of this article. During the Noise 09 experiment, ${ }^{16,17}$ the Research Vessel "New Horizon" was used as an acoustic source; however, there were also periods of time during which no ships were in the vicinity of the arrays. One of these "quiet" periods of time (February 6, 5:00 UTC) coincided with good wind speed and wave heights, and has been used to provide the result shown in Fig. 3(b), using Harrison and Simons's original technique. The plot displays a critical angle around $30^{\circ}$, above which bottom-loss levels between 7 and $8.3 \mathrm{~dB}$ are estimated, with the highest values concentrated at the higher frequencies. The plot also shows how the characteristics of the array influence the estimate: The $1 \mathrm{~m}$ spacing causes the onset of grating lobes at the design frequency of $750 \mathrm{~Hz}$. These disrupt the bottom loss above $700 \mathrm{~Hz}$, limiting the estimate to relatively low frequencies. At these frequencies, the angular resolution of the array is relatively low, and striations due to bottom layering may be "blurred" in the bottom-loss plot. The degradation of the angular resolution with decreasing
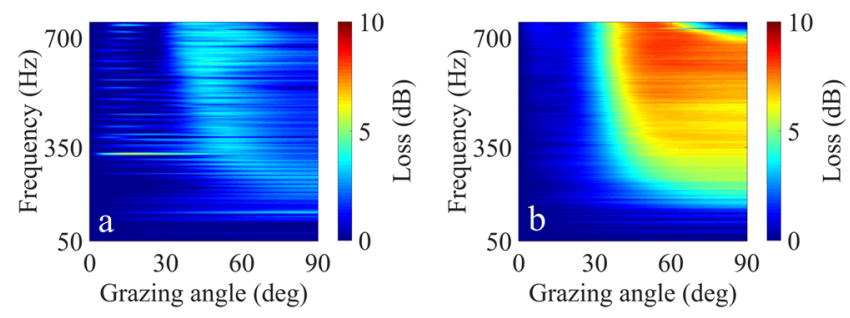

FIG. 3. (Color online) Noise 09: Bottom loss estimated by Harrison and Simons's technique over an average of $10 \mathrm{~min}$ on January 31 (a) and February 6 (b). The significantly underestimated bottom loss visible in (a) when compared to (b), is due to the lower sea state during data acquisition. This figure shows that, during this event, the natural surface noise was not high enough to generate bottom-loss values near those shown in (b). The "arc" visible in (a) at the location where the critical angle appears in (b), is due to the Research Vessel New Horizon, present in the vicinity of VLA1 during the data collection, but at such a distance that its arrival at the array falls below the critical angle. The bottom-loss estimate in (b), obtained from the beamformer output shown in Fig. 2(a), corresponds to a period of absence of loud artificial sources in the vicinity of VLA1, and is used as the reference in this study. 
frequency also causes the widening of the high-level area on the left of the beamformer-output plots in Figs. 2(a) and 2(c): Below $200 \mathrm{~Hz}$, leakage from directions other than the steering angle causes the measured output to become symmetric with respect to the horizontal. In these conditions, the ratio in Eq. (2) approaches unity, and this technique cannot estimate bottom loss.

\section{Theoretical and practical considerations}

The first consideration relevant to the treatment that follows is a peculiar property of the surface-noise field. The theoretical proof of the technique models the surface noise as a sheet of (uniformly distributed) incoherent point sources at the surface. When computing the coherence function between two sensors, an integral is carried out over the range variable $r$, summing the contributions of annular surface elements of radial extent $d r$. The original proof by Harrison shows that, as $r$ increases, the increase in the area of the annular region compensates exactly the loss due to geometric spreading; in other words, the surface-noise field is not affected by geometric spreading, a property that clearly does not apply in the case of a discrete source, such as a ship.

The second important consideration is the VLA's azimuthal ambiguity: A beamformer applied to this type of array cannot identify the azimuth coordinate $\phi$ of a source. When steered toward a certain angle $\vartheta$, it will harvest energy from the entire cone of semiaperture $\left(90^{\circ}-\vartheta\right)$ and axis coinciding with the array axis. In other words, at each steering angle $\vartheta$ the VLA beamformer performs an integration over the whole $360^{\circ}$ of the azimuth coordinate $\phi$. For the purpose of this study, the important consequence of this ambiguity is that the actual distribution of the sources in the annular region has no influence on the beamformer's output, which will be the same regardless of whether, e.g., the same sources are concentrated in a small sector of area $r d r d \phi$, rather than uniformly distributed in the entire annular region.

The considerations illustrated in Sec. III B can be exploited to devise a procedure for processing the ship noise - prior to beamforming — in such a way that it presents characteristics that are similar to those of natural surface noise. In the remainder of this article, the array is assumed to be moored to the bottom, and the angle between the midpoint of the array and the position of the acoustic source (e.g., a ship) will be designated as the source elevation, with the $90^{\circ}$ elevation corresponding to the ship being directly above the array. The idea at the basis of the technique proposed in this article is to build the CSD matrix by summing the contribution of the ship noise measured along the entire trajectory. Assuming for the sake of simplicity that the source level of the wind/wave noise at the surface is too low to produce any measurable bottom loss, the ship is the only available source, and the bottom loss can only be estimated at angles at the receiver corresponding to elevation angles that exist in the actual trajectory of the ship. For the sake of simplicity, in the remainder of this article the ship trajectory will be assumed to be a straight line, and the directivity pattern of this source will be ignored.
Although the average shown in Eq. (5) is adequate when applying Harrison and Simons's technique to natural surface-noise data, the same procedure results in visibly erroneous bottom-loss estimates in the case of ship-noise data (an example using measured data is shown in Sec. IV). This is due to the different physical nature of the two sources: Since the natural-noise source extends indefinitely over the sea surface, when carrying out the average in Eq. (5) the data are sampling the field at all values of $\theta_{r}$ simultaneously. Furthermore, as illustrated above, the integrated contribution of all these arrivals is not affected by attenuation due to geometric spreading.

Ship noise, however, does undergo spreading attenuation, and arrivals at different $\theta_{r}$ will be affected differently, because they correspond to different path lengths between source and receiver. If these differences are not accounted for while averaging the CSD matrix, the spatial stationarity of the coherence function does not hold, and the basic assumptions of the technique are violated.

\section{Ship-noise preprocessing}

In order to produce a CSD matrix that is amenable to processing by Harrison and Simons's technique, in the case of ship noise one must devise a way of restoring the characteristics that are typical of natural-noise CSD matrices. The first step toward this goal is dividing the ship trajectory into segments; the generic $n$th segment sees the ship cover a certain (small) range interval $\Delta r_{n}$, corresponding to an elevation interval $\Delta \theta_{n}$. The acoustic data from each segment are used to produce a segment CSD matrix, which is then normalized to remove the effects of geometric spreading. The normalized segment CSD matrices are then summed to compute the cumulative CSD matrix, containing contributions from all elevation angles available for the given ship trajectory.

In this study, the generic element $c_{i, j}$ of the CSD matrix is normalized by computing the normalized form of the coherence function $C_{\omega}^{\prime}\left(\mathbf{r}_{i}, \mathbf{r}_{j}\right)$, as defined in the treatment by Deane et al. ${ }^{24}$

$$
C_{\omega}^{\prime}\left(\mathbf{r}_{i}, \mathbf{r}_{j}\right)=\frac{C_{\omega}\left(\mathbf{r}_{i}, \mathbf{r}_{j}\right)}{\sqrt{C_{\omega}\left(\mathbf{r}_{i}, \mathbf{r}_{i}\right) C_{\omega}\left(\mathbf{r}_{j}, \mathbf{r}_{j}\right)}} .
$$

By dividing each element of the segment CSD matrix by the square root of the product of the power spectral densities at the sensors, this process "scales" it so that the cumulative CSD matrix is not dominated by the contribution of those segments in the trajectory where the ship is closest to the array. It is noted here explicitly that, due to the azimuthal ambiguity of the VLA beamformer, the fact that the ship only generates contributions to the field from a limited azimuth interval, rather than distributing the same amount of energy uniformly over an annular region around the array, does not alter the array output, and therefore does not impact the applicability of this technique. Furthermore, in the original processing done with Harrison and Simons's technique, the normalization is not strictly required, and carrying it out on the segment CSD matrix or on the cumulative CSD matrix yields virtually equivalent bottom-loss estimates. 


\section{RESULTS AND DISCUSSION}

\section{A. NOISE 09 data}

The results of processing natural surface noise from Noise $09^{16,17}$ data have been shown in Sec. III and will be used in this section as a reference to assess the results produced by the technique proposed in this article. For the shipnoise estimate of the bottom loss, an event has been chosen from the dataset (January 31, $41 \mathrm{~min}$ of data starting at 01:35:00 UTC), during which a ship moves between a minimum range of $51 \mathrm{~m}$ and a maximum of $3544 \mathrm{~m}$ from VLA1. The spectrogram and elevation (deduced from GPS data of the vessel position) are shown in Fig. 4 for the entire duration of the event. Note that, due to uncertainty in the position of VLA1, the time of the closest point of approach does not coincide perfectly in the plots.

The bottom-loss estimates shown later in this section have been obtained by analyzing the first $600 \mathrm{~s}$ of the trajectory whose data are illustrated in Fig. 4, which cover elevations down to about $8^{\circ}$ (the maximum elevation is more uncertain, as the plot in Fig. 4 would seem to indicate a value around $70^{\circ}$ but the estimated bottom loss seems to cover higher angles). The ship trajectory has been divided into $60 \mathrm{~s}$ segments; within each segment, the $\mathbf{p}_{n}(\omega)$ vectors of Eq. (5) are created by taking the Discrete Fourier Transform of $2^{13}$ sample data snapshots; the outer products are then averaged as in Eq. (5), and the resulting CSD matrix is normalized according to Eq. (6). The cumulative CSD matrix (obtained by summing all the individual segment CSD matrices, and dividing the result by the total number of segments) is then beamformed and the beamformer output used to estimate the bottom loss, as shown in Eqs. (1) and (2) above.

As discussed in Sec. III, while the normalization is not strictly required when processing natural surface noise, the case of ship noise is different, due to the discrete nature of the source. To show how critical it is to normalize the segment CSD matrices prior to beamforming, Fig. 5(a) shows the bottom-loss estimate obtained by normalizing a cumulative CSD matrix built from unnormalized segment CSD matrices. In the case of natural surface noise, this choice would not affect the bottom-loss estimate appreciably, but a comparison with Fig. 3(b) shows that the plot in Fig. 5(a) presents an abnormally high loss at the lower frequencies
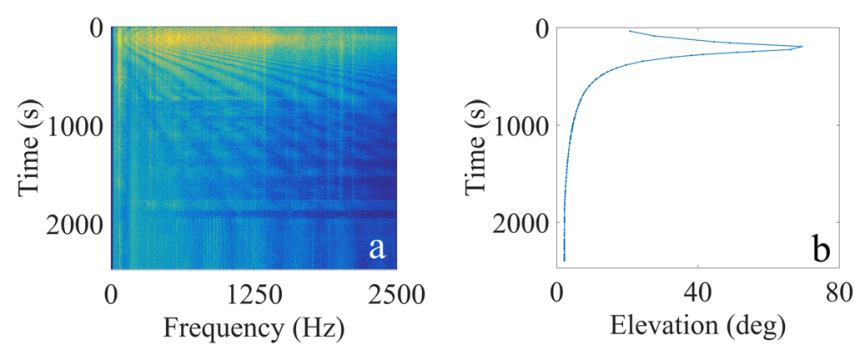

FIG. 4. (Color online) Noise 09 data. Ship event of January 31: Spectrogram from element \#5 (a) and elevation angle (b) for the event recorded by VLA1 used to estimate the bottom loss. The elevation is estimated assuming the array has the shallowest sensor at $7 \mathrm{~m}$ from the seabed, in a $150 \mathrm{~m}$ water column, and the array position as given in the Noise $09 \log$. Due to uncertainty in the position of the array, the times of the closest point of approach do not coincide exactly in the two plots.
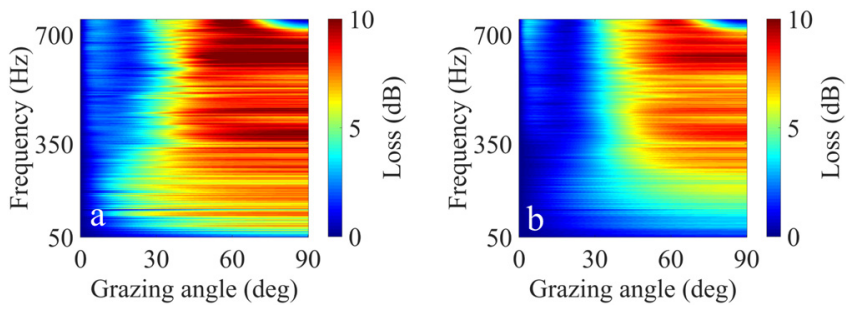

FIG. 5. (Color online) Noise 09 data, ship event of January 31. (a) Bottom loss estimated from ship noise over the first 10 min of the trajectory shown in Fig. 4, normalizing only the cumulative CSD matrix at the end of the average. At the lower frequencies, the plot shows an abnormally high loss and a critical-angle shift (toward lower values) that is not supported by the reference result in Fig. 3(b). (b) Bottom loss estimated from the same data as in (a), but normalizing the CSD matrix over 1 min segments. The plot appears very similar to the natural-noise reference in Fig. 3(b), in terms of critical-angle position, although the level is a bit higher in this case. The area of non-zero bottom loss at the shallowest angles, particularly visible in the high-frequency range, is likely due to lack of ship data at the corresponding ship elevations.

and a critical-angle shift toward lower values that are not supported by the reference result.

On the other hand, the bottom loss estimated from shipnoise data by normalizing each segment CSD matrix is shown in Fig. 5(b). This plot is quite similar to the natural-noise-only estimate of Fig. 3(b), particularly in the critical-angle location and in the higher-loss area in the high-frequency region. The bottom-loss level appears to be a bit higher than in the natural noise case; it is unclear whether this is an artifact, or it is due to insufficient wave noise at the surface for the estimate in Fig. 3(b). It is interesting to note that no particular "features" appear at the steepest angles, where the elevation plot in Fig. 4 would seem to indicate no ship noise could originate. This can in part be due to the width of the beams at these relatively low frequencies, or could be due to the array actual position being different from that of the deployment site, resulting in covering of steeper angles than the deployment location would allow.

The region of higher loss visible at very low grazing angles (particularly at the high end of the frequency range) is likely due to the lack of averaging at the shallowest angles. In the case of ship noise, obtaining bottom loss at these angles can be particularly difficult, due to the very long "tail" visible in the elevation plot in Fig. 4. At the shallowest angles, the ship is far enough for its signal to be attenuated significantly, when it reaches the array. If the measurable signal gets too close to the acquisition-system self-noise, the bottom loss can be significantly underestimated. Furthermore, the normalization, which we have seen is important in removing the effects of geometric spreading, can enhance the contribution of "selfnoise" CSD matrices to the cumulative CSD matrix, impairing the estimate at all angles. The plot in Fig. 3(a) can help substantiate the result in Fig. 5(b), and better illustrate the disruptive effect of including normalized self-noise in the CSD average. Figure 3(a) was obtained by the same procedure as described above, but applied to the last $600 \mathrm{~s}$ of the ship trajectory. In this case, the ship is at a range corresponding to elevations below the critical angle, and the only visible contributions to the bottom loss are along the left margin of the "higher loss" area visible in the natural-noise-only case shown in Fig. 3(b). This shows that, during these $10 \mathrm{~min}$, the natural 
surface noise was not high enough to generate the bottom-loss values shown in Fig. 3(b) (obtained 6 days later), supporting the conclusion that the estimate in Fig. 5(b) is due to the ship noise. Evidently, the addition of CSD matrices such as those that produced the result in Fig. 3(a) would have a disruptive effect on the bottom-loss estimate.

\section{B. Ship simulation}

In this section, simulation is used to illustrate how the preprocessing technique proposed in this article modifies the structure of the CSD matrix. For this example, the watercolumn and bottom properties (shown in Table I), as well as the array spacing, have been chosen so as to allow a direct comparison to the results obtained from experimental data, shown in Sec. IV A. In this study, the tool chosen for simulations is OASN, the surface-noise module of the OASES package. $^{25}$ OASN simulates propagation in horizontally stratified, range independent media by solving the wave equation through the wavenumber-integration method, and can be used to produce directly either the (complex) pressure field over a two-dimensional geometric grid, or the CSD matrix of an array at an arbitrary location in space. In this example, the OASN built-in surface-noise source is used to produce the CSD matrix of a 20-element array, whereas a point source with a flat spectrum divided in $15 \mathrm{~Hz}$ bins in the interval $5-500 \mathrm{~Hz}$, and placed at a depth of $0.75 \mathrm{~m}$ (i.e., $3 / 4$ of the wavelength corresponding to the highest frequency of the source), is used to produce the complex pressure field at $1 \mathrm{~m}$ intervals along a radial trajectory, between $1 \mathrm{~m}$ and the desired maximum range from the source. In both cases, the water column is sampled at $1 \mathrm{~m}$ intervals between the surface and the bottom, located at $150 \mathrm{~m}$ of depth, and a 20-element array is extracted by choosing data from 20 consecutive points, between the depths of 116 and $135 \mathrm{~m}$.

In this exercise, a ship is assumed to move toward the array over a rectilinear trajectory, starting at $1555 \mathrm{~m}$ from the array and ending right above it, at a speed of $1.25 \mathrm{~m} / \mathrm{s}$. The trajectory is "sampled" every $1 \mathrm{~s}$, and subdivided into non-overlapping segments, each corresponding to a duration of $30 \mathrm{~s}$. A segment CSD matrix is created by extracting the values of the precomputed complex field at the array sensor depths, and at the ranges covered by the ship in the segment; the outer products of the data vector are then summed as in Eq. (5) (in this case, $N$ is the number of discrete range values available for the current segment in the precomputed field), and the matrix is normalized according to Eq. (6).

To show how the processing outlined above achieves the intended goal, Fig. 6 shows the real part of the normalized CSD matrix generated by the surface-noise source in

TABLE I. Water-column and bottom configuration for the simulated case; $\Delta$ is the layer thickness, $c_{p}$ is the sound speed, $\rho$ is the density, $\alpha_{c}$ is the compressional volume attenuation, and $\lambda$ is the wavelength.

\begin{tabular}{lcccc}
\hline \hline & $\Delta(\mathrm{m})$ & $c_{p}(\mathrm{~m} / \mathrm{s})$ & $\rho\left(\mathrm{kg} / \mathrm{m}^{3}\right)$ & $\alpha_{c}(\mathrm{~dB} / \lambda)$ \\
\hline Water & 150 & 1500 & 1000 & $1 \times 10^{-4}$ \\
Bottom & $\infty$ & 1700 & 2000 & 0.5 \\
\hline \hline
\end{tabular}

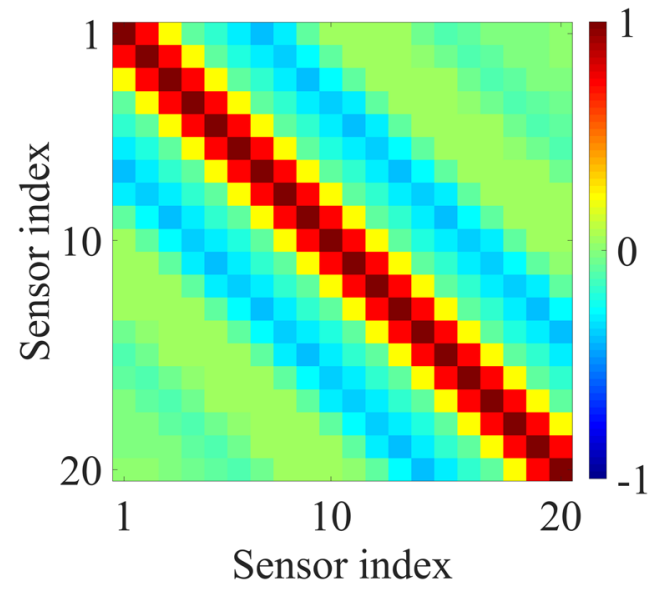

FIG. 6. (Color online) Simulation: Real part of the CSD matrix at $395 \mathrm{~Hz}$, created by OASN's (natural) surface-noise source for the bottom described in Table I. Similar to the data-derived case shown in Fig. 2(b), the CSDM generated by surface noise shows the expected quasi-Toeplitz structure.

OASN (displaying the expected quasi-Toeplitz structure), and Fig. 7 shows the real part of both the segment and the cumulative CSD matrices, at different moments along the trajectory. For the sake of clarity, it is noted here explicitly that the cumulative matrices in Fig. 7 result from the sum of all the segments along the trajectory up to the one for which the corresponding segment matrix is displayed, whereas in the rest of this article the adjective "cumulative" designates a sum taken over the entire trajectory of the ship. While the segment CSD matrices in Fig. 7 do not show a Toeplitz structure, due to the localized nature of the discrete source, as the ship moves along the trajectory (thereby adding new angles of arrival at the receivers) the cumulative CSD matrix progressively reaches a quasi-Toeplitz structure very similar to that created by OASN's surface-noise source [compare Figs. 7(f) and 6].

Finally, Fig. 8 shows the bottom loss estimated from the OASN data used to produce Figs. 6 and 7(f). The two results compare in terms that are very similar to those of the comparison between Figs. 3(b) and 5(b): The ship-noise based result appears noisier, but there is good agreement in critical angle and overall loss values.

\section{CONCLUDING REMARKS}

A technique is proposed that extends the applicability of Harrison and Simons's method for passive bottom-loss estimation to cases in which a ship is moving in the vicinity of the array. The presence of a discrete source, such as a ship, disrupts the spatial stationarity of the noise coherence function, which is necessary for Harrison and Simons's technique to produce accurate results. However, by subdividing the time history recorded at the sensors into segments, computing and normalizing a CSD matrix for each segment, and then averaging such matrices over the entire duration of the event, the resulting data present features similar to those of natural surface noise and therefore become amenable to the original technique. The proposed technique has been applied to data from the Noise 09 experiment, showing that, when 

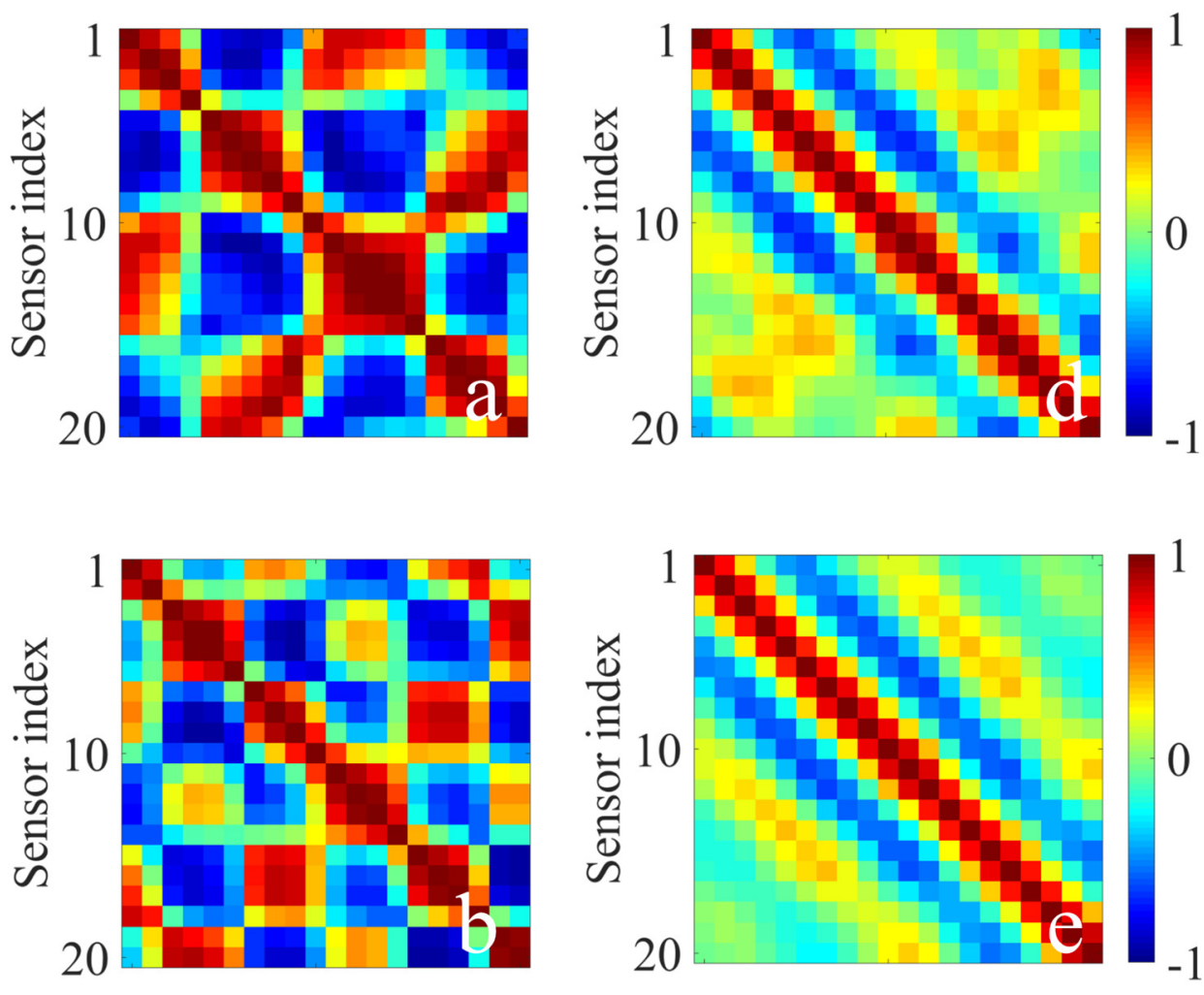

FIG. 7. (Color online) Ship simulation: Real part of CSD matrices at $395 \mathrm{~Hz}$. (a)-(c): Segment CSD matrix for segment \#8 (range 1294-1257 m), \#20 (844-807 m), and \#41 (57-20 m). (d)-(f): Cumulative CSD matrix, created summing the segment CSD matrices up to the corresponding one on the left side. The cumulative CSD matrix for segment \#41 (the last one in this trajectory) clearly approaches the natural-noise-only reference (see Fig. 6).
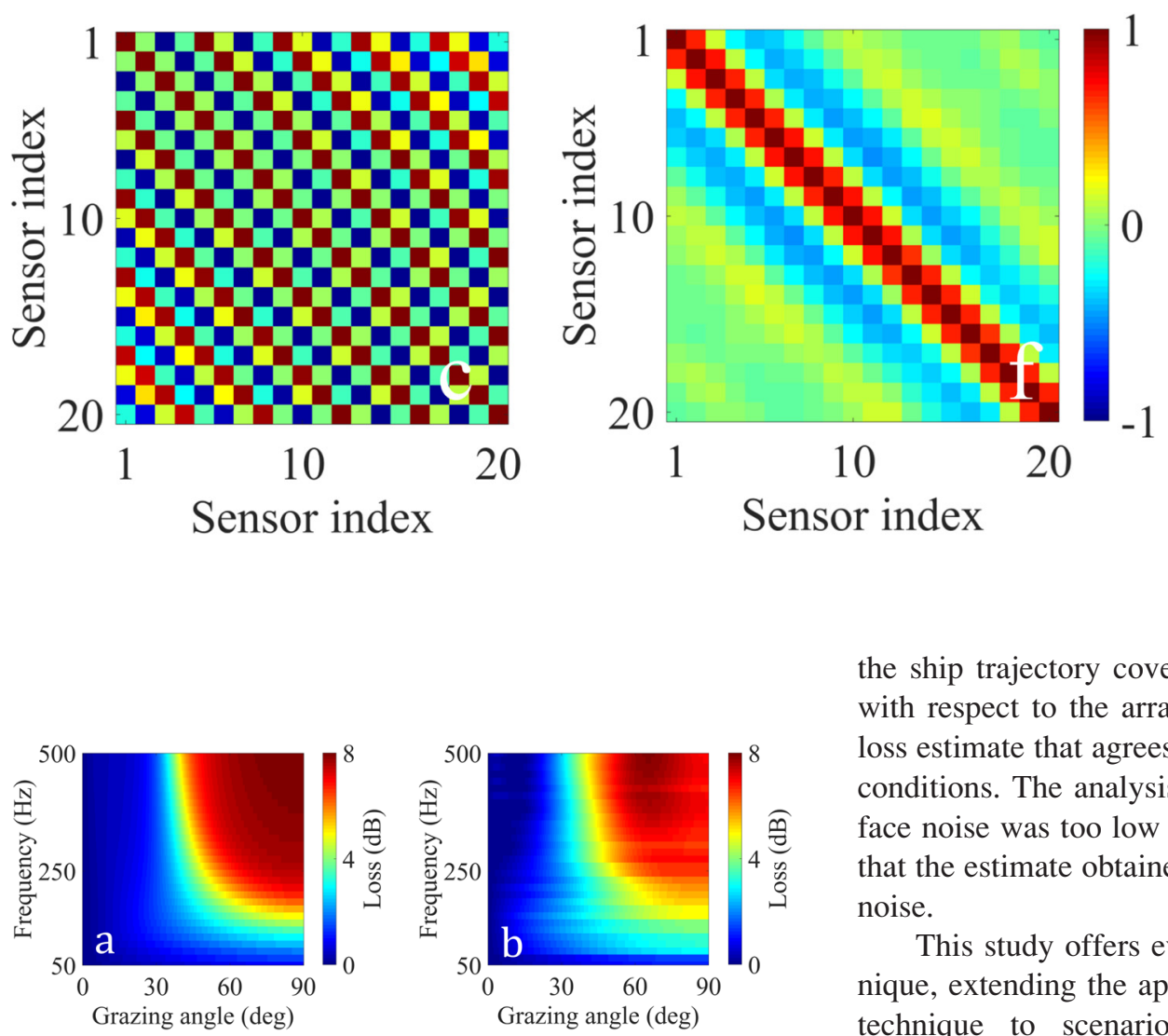

the ship trajectory covers a wide range of elevation angles with respect to the array, it is possible to obtain a bottomloss estimate that agrees with those obtained in natural-noise conditions. The analysis of a case in which the natural surface noise was too low to produce measurable loss confirms that the estimate obtained by the technique is due to the ship noise.

This study offers evidence of the potential of this technique, extending the applicability of Harrison and Simons's technique to scenarios of low natural surface noise. However, new areas now become open to further investigation, among which the possibility of averaging data from more than a single ship trajectory, and a more systematic definition of the data-segmentation algorithm (considering for example the effects of ship velocity and array depth) appear particularly promising. 


\section{ACKNOWLEDGMENTS}

The authors would like to thank William Hodgkiss (Chief Scientist) and Dave Ensberg at the Marine Physical Laboratory, Scripps Institution of Oceanography, University of California, San Diego for providing the Noise 09 experimental data. The authors would also like to gratefully acknowledge the Office of Naval Research Ocean Acoustics for supporting this research under the Multidisciplinary University Research Initiative, "Exploitation of Natural and Anthropogenic Noise for Ocean Exploration."

${ }^{1}$ C. Ferla and F. B. Jensen, "Are current environmental databases adequate for sonar predictions in shallow water?," in Impact of Littoral Environmental Variability on Acoustic Predictions and Sonar Performance, edited by N. G. Pace and F. B. Jensen (Springer Science+Business Media, Dordrecht, the Netherlands, 2002), pp. 555-562.

${ }^{2}$ R. A. Koch and D. P. Knobles, "Geoacoustic inversion with ships as sources," J. Acoust. Soc. Am. 117, 626-637 (2005).

${ }^{3}$ S. E. Crocker, P. L. Nielsen, J. H. Miller, and M. Siderius, "Geoacoustic inversion of ship radiated noise in shallow water using data from a single hydrophone," J. Acoust. Soc. Am. 136, EL362-EL368 (2014).

${ }^{4}$ D. J. Battle, P. Gerstoft, W. A. Kuperman, W. S. Hodgkiss, and M. Siderius, "Geoacoustic inversion of tow-ship noise via near-field-matchedfield processing," IEEE J. Ocean. Eng. 28, 454-467 (2003).

${ }^{5}$ C. Park, W. Seong, and P. Gerstoft, "Geoacoustic inversion in time domain using ship of opportunity noise recorded on a horizontal towed array," J. Acoust. Soc. Am. 117, 1933-1941 (2005).

${ }^{6}$ D. Tollefsen and S. E. Dosso, "Bayesian geoacoustic inversion of ship noise on a horizontal array," J. Acoust. Soc. Am. 124, 788-795 (2008).

${ }^{7}$ N. R. Chapman, R. M. Dizaji, and R. L. Kirlin, "Inversion of geoacoustic model parameters using ship radiated noise," in Acoustic Sensing Techniques for the Shallow Water Environment (Springer, the Netherlands, 2006), pp. 289-302.

${ }^{8}$ C. Gervaise, B. G. Kinda, J. Bonnel, Y. Stéphan, and S. Vallez, "Passive geoacoustic inversion with a single hydrophone using broadband ship noise," J. Acoust. Soc. Am. 131, 1999-2010 (2012).

${ }^{9}$ C. H. Harrison and D. G. Simons, "Geoacoustic inversion of ambient noise: A simple method," J. Acoust. Soc. Am. 112, 1377-1389 (2002).
${ }^{10}$ M. Siderius and C. Harrison, "High-frequency geoacoustic inversion of ambient noise data using short arrays," AIP Conf. Proc. 728, 22-31 (2004).

${ }^{11}$ C. H. Harrison, "Sub-bottom profiling using ocean ambient noise," J. Acoust. Soc. Am. 115, 1505-1515 (2004).

${ }^{12}$ J. E. Quijano, S. E. Dosso, J. Dettmer, L. M. Zurk, M. Siderius, and C. H. Harrison, "Bayesian geoacoustic inversion using wind-driven ambient noise," J. Acoust. Soc. Am. 131, 2658-2667 (2012).

${ }^{13}$ P. L. Nielsen, M. Siderius, and L. Muzi, "Performance assessment of a short hydrophone array for seabed characterization using natural-made ambient noise," J. Acoust. Soc. Am. 136, 2155 (2014).

${ }^{14}$ J. I. Arvelo, "Robustness and constraints of ambient noise inversion," J. Acoust. Soc. Am. 123, 679-686 (2008).

${ }^{15}$ L. Muzi, M. Siderius, J. Quijano, and S. Dosso, "High-resolution bottomloss estimation using the ambient-noise vertical coherence function," J. Acoust. Soc. Am. 137, 481-491 (2015).

${ }^{16}$ S. W. Lani, K. G. Sabra, W. S. Hodgkiss, W. A. Kuperman, and P. Roux, "Coherent processing of shipping noise for ocean monitoring," J. Acoust. Soc. Am. 133, EL108-EL113 (2013).

${ }^{17}$ C. M. Verlinden, J. Sarkar, W. S. Hodgkiss, W. A. Kuperman, and K. G. Sabra, "Passive acoustic source localization using sources of opportunity," J. Acoust. Soc. Am. 138, EL54-EL59 (2015).

${ }^{18}$ F. B. Jensen, W. A. Kuperman, M. B. Porter, and H. Schmidt, "Fundamentals of ocean acoustics," in Computational Ocean Acoustics, 2nd ed. (Modern Acoustics and Signal Processing) (Springer, New York, 2011), Chap. 1, pp. 38-50.

${ }^{19}$ C. H. Harrison, "Formulas for ambient noise level and coherence," J. Acoust. Soc. Am. 99, 2055-2066 (1996).

${ }^{20}$ M. J. Buckingham, "A theoretical model of ambient noise in a low-loss, shallow water channel,” J. Acoust. Soc. Am. 67, 1186-1192 (1980).

${ }^{21}$ W. S. Liggett and M. J. Jacobson, "Noise covariance and vertical directivity in a deep ocean,” J. Acoust. Soc. Am. 39, 280-288 (1966).

${ }^{22}$ D. R. Barclay and M. J. Buckingham, "Depth dependence of wind-driven, broadband ambient noise in the Philippine Sea," J. Acoust. Soc. Am. 133, 62-71 (2013).

${ }^{23}$ J. D. Skinner and W. S. Hodgkiss, "A networked/autonomous receiving array system," in IEEE OCEANS. Available at http://dx.doi.org/10.1109/ oceans.2008.5151902 (2008) (Last viewed June 5, 2017).

${ }^{24}$ G. B. Deane, M. J. Buckingham, and C. T. Tindle, "Vertical coherence of ambient noise in shallow water overlying a fluid seabed," J. Acoust. Soc. Am. 102, 3413-3424 (1997).

${ }^{25} \mathrm{H}$. Schmidt, OASES Version 3.1 User Guide and Reference Manual, Massachusetts Institute of Technology, Cambridge, MA (2004). 